\title{
Selection of plant species for permanent ground cover in vineyards: Looking for an agronomic and environmental optimum
}

\author{
N. Delabays ${ }^{1}$, A.F. Grogg $^{2}$, M. Mota $^{3}$ et U. Piantini ${ }^{2}$ \\ ${ }^{1}$ Institute Earth-Nature-Environnement, hepia, University of Applied Sciences of Western Switzerland, 1254 Jussy, Switzerland \\ ${ }^{2}$ Institute of life technologies, hevs, University of Applied Sciences of Western Switzerland, 1950 Sion, Switzerland \\ ${ }^{3}$ Institute of viticulture and œnology, Changins, University of Applied Sciences of Western Switzerland, 1260 Nyon, Switzerland
}

\begin{abstract}
The installation of a permanent ground cover in vineyards offers numerous agronomic (soil protection, soil fertility) and environmental (reduced leaching of nutrients and of plant protection products, reduced use of herbicides, biodiversity) benefits. Nevertheless, such ground covers are not always free of drawbacks (competition for water and nitrogen, increased risk of frost, management). For the growers, the challenge is to manage the green ground covers in such a way as to preserve their advantages while limiting these drawbacks. Among the tools available to the winegrower is the sowing of a seed mixture composed of selected species: a choice depending of the soil and climatic conditions of the parcel, but also of the different, and sometime contradictory, objectives of the grower. This paper lists the agronomic and environmental issues addressed by the installation of a permanent ground cover in vineyards. It describes two concrete situations ground cover for vineyards integrated in agro-ecological networks and green cover on the row of vines (as alternative to glyphosate) - for which, according to the objectives and the impacts, a choice of plant species is proposed. At last, it presents the trials now carried out to validate and optimize those selections, as well as the first observations and results gathered to date.
\end{abstract}

\section{Introduction}

L'installation d'un couvert végétal permanent dans les vignes présente des avantages aujourd'hui bien documentés: protection du sol, amélioration de sa portance et de son activité biologique, diminution du lessivage des substances nutritives et des produits phytosanitaires, réduction de l'usage des herbicides. Parallèlement, un tel couvert participe à la promotion de la biodiversité, y compris fonctionnelle, dans l'agrosystème; et il peut même parfois contribuer à la conservation de plantes menacées.

Ces couvertures ne sont cependant pas toujours exemptes de désavantages: compétition pour l'eau et l'azote, avec ses effets négatifs sur la qualité de la vendange, entretien, risques de gel accrus. En Suisse, ces défauts ont limité l'extension des zones enherbées, et pourraient même les réduire à l'avenir, suite aux évolutions climatiques (sécheresse estivale).

L'installation et l'entretien d'un couvert végétal dans les vignobles doit donc être géré de manière à conserver ses avantages tout en limitant ces inconvénients. Parmi les outils à disposition du viticulteur figure le semis du couvert avec un choix ciblé d'espèces [1] ; un choix complexe, car les plantes constituant le mélange visent des objectifs en partie contradictoires : d'un côté, elles doivent être peu vigoureuses, afin de réduire le nombre de fauches nécessaires à l'entretien de la parcelle, et peu compétitives, pour limiter les interférences avec la culture et, parfois, permettre l'expression d'une flore diversifiée ; d'un autre côté, elles se doivent d'être pérennes, et capables de maîtriser le développement d'adventices banales et agressives.

L'enjeu est encore plus complexe pour l'enherbement sous le rang de culture (cavaillon) : les effets de la compétition y sont exacerbés et, techniquement, les interventions entre les ceps sont plus délicates.

Ce papier propose une synthèse des objectifs, souvent contradictoires, attendus des couverts végétaux ; il expose la priorisation de ses objectifs effectuée dans 2 cas concrets et décrit le choix d'espèces préconisées pour ces derniers; il présente enfin succinctement les expérimentations mises en place pour valider ces choix, ainsi que leurs premiers résultats.

\section{L'enherbement des vignes: des avantages et des contraintes}

Nous avons brièvement mentionné dans notre introduction les divers avantages que peut offrir un couvert végétal permanent, ainsi que ses défauts possibles. Le Tableau 1 présente une liste des différents impacts potentiels d'une telle couverture. L'importance de ces divers éléments variera selon les conditions pédoclimatiques (type de sol, précipitations, zones gélives, pente) et agronomique (cépage, mode de conduite, outillage) : de fait, pratiquement chaque parcelle devrait faire l'objet d'une analyse ciblée.

Ensuite, le viticulteur dispose de quelques outils pour optimiser son couvert, c'est-à-dire promouvoir et 
Tableau 1. Avantages et inconvénients, au niveau du sol, de la culture et de l'environnement, pouvant résulter de l'installation d'une couverture végétale permanente dans une vigne.

\begin{tabular}{|c|c|c|}
\hline & Avantages & Impacts négatifs potentiels \\
\hline \multirow[t]{4}{*}{ Sol } & Réduction de l'érosion & Formation d'un feutrage en surface \\
\hline & Amélioration de la structure et de la portance & \\
\hline & Fixation de l'azote atmosphérique & \\
\hline & Activité biologique (fertilité) & \\
\hline \multirow[t]{3}{*}{ Entretien } & & Fauches \\
\hline & & Glissade (parcelle en pente) \\
\hline & & Pérennité (maintien du couvert) \\
\hline \multirow[t]{2}{*}{ Vigne } & Maîtrise d'une vigueur excessive & Risque de gel accru \\
\hline & & $\begin{array}{l}\text { Compétition hydro-azotée } \\
\text { (qualité de la vendange) }\end{array}$ \\
\hline \multirow[t]{2}{*}{ Phytosanitaire } & $\begin{array}{l}\text { Promotion de la biodiversité fonctionnelle } \\
\text { (auxiliaires) }\end{array}$ & $\begin{array}{l}\text { Maladies (micro-climat dans } \\
\text { la zone des grappes) }\end{array}$ \\
\hline & & $\begin{array}{l}\text { Epidémiologie (hôte de vecteurs et/ou de maladies) } \\
\text { Hôte de ravageurs }\end{array}$ \\
\hline \multirow[t]{5}{*}{ Environnement } & Réduction des herbicides & Développement de plantes envahissantes \\
\hline & $\begin{array}{l}\text { Réduction des lessivages } \\
\text { (phytosanitaires et nutriments) }\end{array}$ & \\
\hline & Promotion de la biodiversité & \\
\hline & Conservation d'espèces & \\
\hline & Fixation carbone & \\
\hline
\end{tabular}

conserver ses avantages, et limiter ses inconvénients; principalement au travers de la fréquence et de l'intensité des fauches. Mais au moment de la décision d'une mise en place d'un couvert permanent, la pratique d'un engazonnement (c'est-à-dire la réalisation d'un semis, par opposition à une simple gestion de la flore spontanée) offre un levier d'optimisation important. Le choix des espèces semées reste cependant complexe, notamment en fonction des contraintes de la parcelle et des objectifs, parfois contradictoires, du vigneron.

Deux exemples concrets vont nous permettre d'illustrer la difficulté, mais également le potentiel, d'un choix ciblé des espèces destinées à l'installation d'un couvert viticole : l'enherbement de vignes à intégrer dans un réseau agro-écologique et l'enherbement du cavaillon (comme alternative au glyphosate).

\section{Exemple 1 : création d'un mélange viticole pour les réseaux agro-écologiques lémaniques}

\subsection{Contexte}

En Suisse, des agriculteurs peuvent s'associer pour établir des réseaux agro-écologiques, dans lesquels des structures de promotion de la biodiversité - jachères florales, prairies extensives, vergers haute-tige - sont installées sur leurs exploitations et reliées entre elles. Parmi ces structures peuvent figurer des 《 vignes à haute diversité naturelle »; mais celles-ci, pour être reconnues, doivent respecter de strictes contraintes qualitatives (composition botanique du couvert) et techniques (régime de fauche limité).

Les mélanges viticoles actuellement disponibles pour enherber les vignes ne permettent souvent pas d'atteindre la qualité botanique minimale requise (qualité I), et ils sont par ailleurs trop vigoureux pour que le vigneron puisse respecter la limitation du nombre de fauches
Tableau 2. Espèces composant le 《mélange pilote lémanique », destiné à l'enherbement de vignes devant répondre aux critères qualitatifs (richesse botanique) et techniques (réduction des fauches) des réseaux agro-écologiques.

\begin{tabular}{|l|l|}
\hline Nom latin & Nom français \\
\hline Achillea millefolium & Achillée millefeuille \\
\hline Agrostis capillaris & Agrostis capillaire \\
\hline Bellis perennis & Pâquerette \\
\hline Bromus tectorum & Brome des toits \\
\hline Lotus corniculatus & Lotier corniculé \\
\hline Medicago lupulina & Luzerne lupuline \\
\hline Origanum vulgare & Origan vulgaire \\
\hline Pimpinella saxifraga & Boucage saxifrage \\
\hline Poa compressa & Pâturin compressé \\
\hline Potentilla neumanniana & Potentille du printemps \\
\hline Prunella vulgaris & Prunelle vulgaire \\
\hline Sanguisorba minor & Petite sanguisorbe \\
\hline Thymus pulgioides & Thym faux pouliot \\
\hline Veronica chamaedrys & Véronique petit-chêne \\
\hline
\end{tabular}

exigée; conséquence : dans de nombreux réseaux, de grandes zones viticole sont exclues, péjorant le potentiel d'amélioration de la biodiversité du milieu agricole.

\subsection{Création d'un mélange pilote}

En 2016, un mélange pilote, potentiellement adapté aux vignobles de la région lémanique, et devant permettre de répondre aux critères des réseaux agro-écologiques, a été développé (Tableau 2).

Il est constitué d'espèces relativement peu vigoureuses, afin de réduire le nombre de fauches nécessaires à l'entretien de la parcelle, et peu concurrentielles, pour limiter les interférences avec la vigne et permettre l'expression d'une flore diversifiée garantissant un niveau de qualité I, voire permettant d'atteindre un niveau 
de qualité II (évalué sur le nombre et l'intérêt des espèces présentes). Ces espèces se doivent cependant d'être pérennes, et capables de limiter le développement d'adventices banales et agressives. Ce choix d'espèces est le résultat d'observations rassemblées dans le cadre d'essais préliminaires, décrits en détails dans une publication antérieure [2].

\subsection{Réseau d'essais et premières observations}

A l'automne 2016 a débuté un projet de validation et d'optimisation de ce 《mélange pilote lémanique»; projet qui vise à confirmer sa pertinence, à préciser, en fonction des conditions édaphiques et agronomiques, sa composition optimale (espèces et proportions), enfin à proposer une gestion adaptée (période et technique de semis, calendrier et intensité des fauches). A cette fin, un réseau de parcelles (25 à ce jour) a été progressivement installé avec ce mélange; parcelles sur lesquelles un suivi régulier de la végétation est planifié sur une période de 3 ans (2017-2019).

Un bilan intermédiaire réalisé fin 2018 a permis de vérifier, notamment en fonction de la période de semis, l'installation et le comportement des différentes espèces du mélange et de préciser la biodiversité qui a pu s'exprimer. Si toutes les espèces du mélange ont été repérées au moins une fois dans l'une ou l'autre des parcelles du réseau, certaines s'expriment dans la majorité des cas (Achillea millefolium, Bromus tectorum, Lotus corniculatus, Medicago lupulina, Prunella vulgaris), alors que d'autres n'apparaissent que très rarement (Pimpinella saxifraga, Potentilla neumanniana, Veronica chamaedrys). A l'exception d'Achillea millefolium, présentant parfois un développement un peu agressif, les autres espèces confirment un comportement intéressant, en particulier une vigueur raisonnable qui permet bien, comparativement aux plantes des mélanges standards, l'expression d'une biodiversité relativement élevée (jusqu'à une cinquantaine d'espèces observées dans les parcelles les plus anciennes du réseau), et également d'envisager une réduction du régime de fauches, de une à 2 coupes par année, en comparaison d'un mélange viticole standard.

Les observations rassemblées à ce jour, sur le développement et l'habitus des espèces du mélange, permettent par ailleurs de confirmer et préciser l'intérêt particulier de plusieurs espèces (Lotus corniculatus, Poa compressa), y compris dans la perspective d'une utilisation pour l'enherbement sur le rang de culture (Medicago lupulina, Prunella vulgaris).

\section{Exemple 2 : sélection d'espèces allélopathiques pour l'enherbement du rang de culture (cavaillon)}

\subsection{Contexte : recherche d'alternatives au glyphosate}

Actuellement, la maîtrise des adventices indésirables dans les vignes repose encore souvent sur l'utilisation d'herbicides de synthèse. En particulier, la gestion du rang de culture - soit la ligne sur laquelle sont plantés les ceps (cavaillon) - reste le principal défi pour une culture sans herbicides; concrètement, aujourd'hui, dans la majorité des parcelles viticoles, c'est le glyphosate qui est utilisé.
Ces applications d'herbicides sont cependant à l'origine de nombreux problèmes, dont l'acuité va croissante: contamination des eaux, développement et dissémination de biotypes d'adventices résistants, perte de biodiversité dans les agroécosystèmes. Ces problèmes, accompagnés de pressions sociétales grandissantes, justifient la recherche de nouvelles stratégies et méthodes de maîtrise des mauvaises herbes, plus écologiques et durables. Les méthodes alternatives classiques, telles la lutte mécanique ou le brûlage, outre qu'elles ne sont ellesmêmes pas exemptes de défauts - forte consommation d'énergies fossiles, risques d'érosion des sols, baisse des taux de matières organiques du sol - offrent par ailleurs des efficacités aléatoires.

\subsection{Potentiel de l'utilisation d'espèces allélopathiques}

Une approche innovante et prometteuse consiste en l'utilisation agronomique des phénomènes d'allélopathie [3] : de nombreuses espèces végétales synthétisent, et relâchent dans l'environnement, des métabolites secondaires capables d'inhiber la germination et la croissance de plantes avoisinantes [4]. Parmi les applications concrètes possibles de ce phénomène figure l'installation, notamment sous le rang de culture, d'un enherbement constitué d'espèces allélopathiques. En effet, cette interaction chimique entre plantes pourrait contribuer à concilier les attentes contradictoires d'un tel couvert : il doit être peu concurrentiel vis-à-vis de la culture, mais néanmoins permettre une maîtrise de la flore adventice agressive et indésirable. A cette fin, c'est à nouveau un choix ciblé d'espèces qui se justifie; en l'occurrence sur la base de critères morphologiques (habitus nanisant et/ou rampant), physiologiques (taux de croissance) et phytochimiques (synthèse de molécules allélopathiques).

\subsection{Le projet NOGLYPHOS}

En juin 2018 a débuté, dans le cadre du programme «Healthfood» de la Haute école spécialisée de Suisse occidentale (HES-SO), un projet intitulé «Alternative au glyphosate : sélection d'écotypes allélopathiques pour une maîtrise durable et écologique des adventices en viticulture et arboriculture». Le but de ce projet est de sélectionner, notamment sur la base de leur traits fonctionnels et de leurs propriétés allélopathiques, des espèces adaptées à l'enherbement du cavaillon. A terme, c'est même la sélections d'écotypes particuliers qui est envisagée : en effet, les espèces proches de l'état sauvage offrent souvent une large diversité aux niveaux morphologiques [5] et de leur composition phytochimique [6].

Dans un premier temps, des biotests de screening ont été développés, afin de détecter les espèces potentiellement allélopathiques. Un de ces biotests est réalisé dans des plaques multi-puits, avec différents substrats (milieu gélosé, sable de silice, sol agricole) dans lesquels des tissus végétaux des plantes testées sont incorporés, avant le dépôt de semences d'une plante cible (Lepidium sativum) (Fig. 1). Sur cette dernière, des mesures du taux de germination et de la croissance des plantules sont ensuite effectués. 


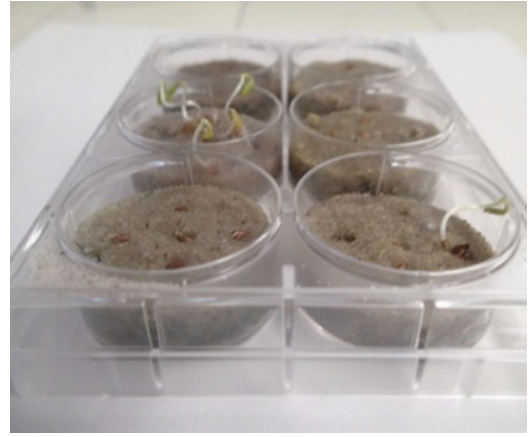

Figure 1. Exemple de biotest, ici sur sable de silice, pour le screening des propriétés allélopathiques d'espèces végétales.

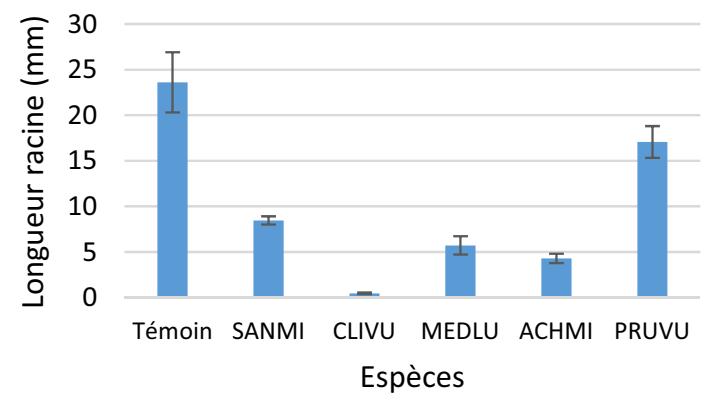

Figure 2. Biotest sur substrat de silice : effets, sur la longueur des racines de cresson alénois, de l'incorporation de feuilles sèches de 5 espèces (moyennes \pm ESM ; $n=4$ ). (SANMI : Sanguisorba minor ; CLIVU : Clinopodium vulgare ; MEDLU : Medicago lupulina ; ACHMI : Achillea millefolium ; PRUVU : Prunella vulgaris).

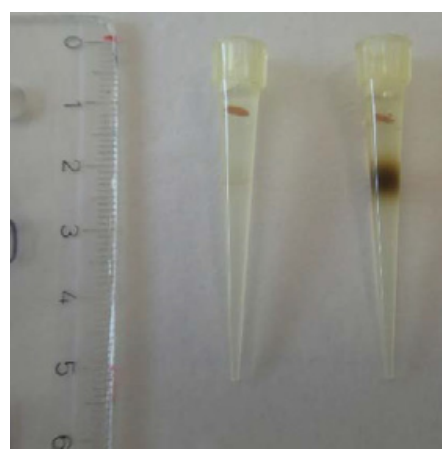

Figure 3. Micro-biotest, de type 《 sandwich », utilisé pour la mise en évidence des propriétés phyto-toxiques d'extraits végétaux, ou de molécules purifiées, disponibles en très petites quantités.

Ces biotests nous ont permis de mettre en évidence les propriétés phyto-toxiques d'espèces telles que Bromus tectorum, Medicago lupulina ou encore Clinopodium vulgare (Fig. 2).

Dans une deuxième étape, ces plantes ont fait l'objet d'extractions et de fractionnements [7] : à chaque étape, les propriétés phyto-toxiques ont été vérifiées, à l'aide d'un micro-biotest effectué sur milieu gélosé, en embouts de pipette (Fig. 3) ; un biotest réalisable avec de très petites quantités d'extraits [7].

L'objectif est de déterminer la ou les molécules impliquées dans les propriétés allélopathiques des espèces détectées dans le cadre des screenings (Fig. 4).

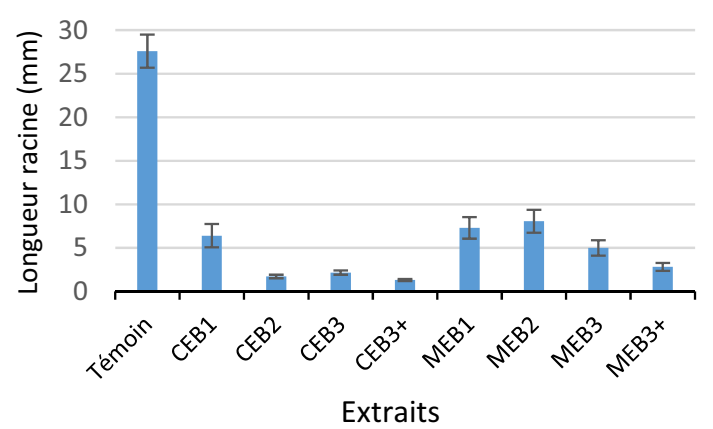

Figure 4. Micro-biotest en embouts : effets, sur la longueur des racines de cresson alénois, de différentes fractions d'extraits végétaux (CEB : Clinopodium vulgare ; MEB : Medicago lupulina) (moyennes $\pm \mathrm{ESM} ; n=18$ ).

A ce jour, 4 métabolites ont été isolés à partir d'une fraction particulièrement phyto-toxique de Bromus tectorum, dont 3 ont pu être identifiés [7].

La détermination de ces molécules ouvre des possibilités d'optimisation de l'utilisation du phénomène d'allélopathie au champ. D'abord, un suivi de la dynamique de production et de relâchement de ces molécules devient envisageable ; une connaissance susceptible de permettre une meilleure gestion du couvert: période de fauche, utilisation d'un mulch ou de résidus, par exemple. Surtout, la connaissance des molécules impliquées facilitera la sélection, à l'intérieur de l'espèce considérée, d'écotypes particulièrement allélopathiques ; en effet, les populations des espèces proches de l'état sauvage offrent souvent une forte variation de leurs teneurs en métabolites secondaires [6].

\section{Conclusion et perspectives}

Relever les défis liés à l'enherbement de sols viticoles, notamment sous le rang de culture, est techniquement très complexe. Outre la prise en compte des conditions pédoclimatiques, très variables, il convient de pondérer et de concilier de nombreux objectifs agronomiques et environnementaux, parfois contradictoires.

Dans cette perspective, le choix des espèces composant cet enherbement est potentiellement un puissant levier à disposition des viticulteurs ; de fait, le mélange optimal ne sera assurément pas le même pour l'enherbement du cavaillon ou des inter-rangs, ou si la parcelle viticole vise à s'inscrire dans un réseau agro-écologique, par exemple.

Aujourd'hui, le choix des espèces se réalise encore souvent de manière empirique. Des expérimentations restent nécessaires pour valider et améliorer les mélanges proposés. Surtout, l'importance et l'urgence des enjeux, comme la conservation de la biodiversité ou le développement d'alternatives au glyphosate, justifient une sélection plus ciblée des espèces et de leurs écotypes, voire la création de lignées adaptées.

\section{Références}

[1] N. Delabays, J.-L. Spring, A. Ançay, E. Mosimann et A. Schmid, Rev suisse Viti. Arboric. Hortic. 32, 322 (2000)

[2] N. Delabays, G. Pétremand, D. Fleury, Rev suisse Viti. Arboric. Hortic. 48, 322 (2016) 
[3] M.M. Trezzi, R.A. Vidal, A.A. Balbinot Junior, H. von Hertwig Bettencourt, A.P. da Silva Souza Filho, J. Plant Interac. 11, 53 (2016)

[4] Z.A. Cheema, M. Farooq, A. Wahid, Allelopathy. Current Trends and Future Applications (Berlin, Springer International Publishing, 2013)

[5] I. Quarta, Création d'un Mélange Optimal de Couverture en Cultures Spéciales Pérennes : Suivi de Parcelles Expérimentales Pilotes et Établissement d'une Collection de Medicago Lupulina (Hepia, Genève, Travail de Bachelor, 2018)

[6] K.R. Khanna, Biochemical Aspects of Crop Improvement (Boston, CRC Press, 1991)

[7] H. Davet, Caractérisation Structurelle des Métabolites Allélopathiques de Bromus tectorum L. (EIValais-Wallis, Sion, Travail de Bachelor, 2018) 